\title{
Patellar Maltracking as a Cause of Lameness in Two Dachshunds
}

\author{
David Rhys Treharne $^{1}$ Neil James Burton ${ }^{1}$ \\ ${ }^{1}$ Wear Referrals, Bradbury, Stockton-on-Tees, United Kingdom \\ VCOT Open 2018;1:e8-e11.
}

\begin{abstract}
Address for correspondence Neil James Burton, BVSc, DSAS(Orth), CertSAS, MRCVS, Wear Referrals, Bradbury, Stockton-on-Tees TS21 2ES, United Kingdom (e-mail: neil.burton@wear-referrals.co.uk).
\end{abstract}

\author{
Abstract \\ Keywords \\ - patellar maltracking \\ - dachshund \\ - chondromalacia \\ patellae
}

Two Dachshunds were presented for the complaint of intermittent unilateral pelvic limb skipping lameness. Clinical examination and radiographs did not reveal any abnormalities. Computed tomography (CT) imaging showed changes in the femoropatellar joint space in both dogs and surgical evaluation revealed femoral trochlear and patellar cartilage loss. Patellar maltracking without patellar luxation or instability was diagnosed. Patellar maltracking should be included as a differential diagnosis for pelvic limb lameness in this breed.

\section{Introduction}

In human beings, anterior knee pain is a common complaint, reported to affect up to one in four members of the general population. ${ }^{1}$ The most common cause of anterior knee pain is functional patellofemoral pain syndrome, ${ }^{2}$ which has an overall prevalence of $6.4 \%$ in the population. ${ }^{3}$ The aetiopathogenesis of functional patellofemoral pain syndrome is multifactorial and includes patellar maltracking, dynamic valgus (that being where the knee collapses medially during stance due to valgus and/or rotation) and peri-patellar muscle imbalance. ${ }^{4}$ In the case of patellar maltracking, which is usually lateral in humans, mal-articulation of the femoropatellar joint may result in focal overload of the patellar cartilage, anterior knee pain and may progress to chondromalacia patellae. Although patellar luxation in dogs is a relatively common orthopaedic disorder, ${ }^{5}$ to the authors' knowledge, patellar maltracking without patellar instability or luxation as a cause of lameness has so far not been described. The case report herein outlines the clinical, diagnostic and surgical findings of two Dachshunds that were presented for the complaint of intermittent skipping lameness due to patellar maltracking.

\section{Case One}

A male Dachshund was presented at 6 months of age with a 1-month history of intermittent left hind skipping lameness received

July 17,2018

accepted

August 9, 2018 with no prior history of trauma. On examination, intermittent left pelvic limb skipping lameness when trotting was present; lameness was not present when walking. Orthopaedic examination was unremarkable and the patellae were palpably reduced in each respective femoral trochlea and palpably stable both medially and laterally throughout stifle range of motion. Radiographs of the pelvic limbs were performed that did not reveal any abnormalities; there was no effusion or osteoarthritic change on orthogonal views of either stifle. Palpation of the pelvic limbs under sedation and a rectal examination did not reveal any abnormalities. A 6-week period of exercise restriction and treatment with meloxicam (Loxicom, Norbrook Laboratories (GB) Ltd., Corby, United Kingdom) was advised with re-examination if signs persisted. Following this 6 -week period, the skipping lameness in this limb had become more frequent. Repeat orthopaedic examination did not reveal any abnormalities bar the skipping lameness when trotting as previously. Computed tomography (CT) of the pelvic limbs was performed (Siemens Somaton Emotion 16 Slice, Siemens Healthcare Limited, Camberly, United Kingdom), both dogs were placed in sternal recumbency with their stifles in a neutral position. The findings of the CT imaging were unremarkable bar the left patella being medially sub-luxated within the trochlear groove with femoropatellar contact predominantly with the axial portion of the medial femoral trochlea with mild sub-patellar sub-chondral sclerosis (-Fig. 1); there was no osteophytosis or joint effusion.
License terms Stuttgart · New York
DOI https://doi.org/

10.1055/s-0038-1672116. ISSN $2625-2325$. 


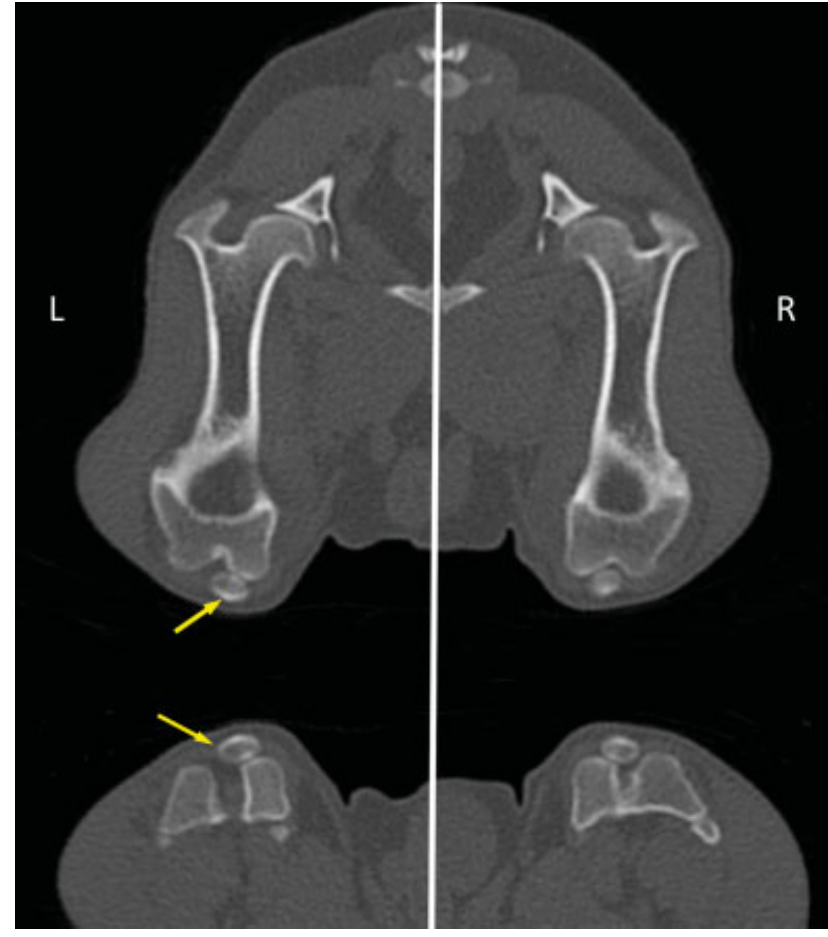

Fig. 1 Dorsal and transverse plane computed tomography (CT) images of the femora and stifles in case 1 . Note that the left patella appears medially sub-luxated within the trochlear groove (yellow arrows) with femoropatellar contact predominantly with the axial portion of the medial femoral trochlea. There is also evidence of left focal sub-patellar sub-chondral sclerosis.

Inspection in the joint via a medial parapatellar arthrotomy revealed mild fibrillation (Outerbridge Grade II) ${ }^{6}$ of the cartilage on the axial portion of the medial aspect of the trochlear groove and on the cartilage on the underside of the body of the patella. Surgery was performed comprising a block recession sulcoplasty, lateral tibial tuberosity transposition, medial retinacular release and lateral imbrication.

\section{Case Two}

An 18-month-old male Dachshund was presented with a 2-month history of progressive left hind skipping lameness with no previous history of trauma. On examination, frequent skipping lameness in the left pelvic limb was present when both walking and trotting. Orthopaedic and neurological examinations were unremarkable. The patellae were reduced in the trochlear grooves and were stable on medial and lateral palpation through stifle range of motion. Palpation of the pelvic limbs under sedation and a rectal examination did not reveal any abnormalities. The referring veterinary surgeon's radiographs showed chondrodystrophoid degeneration of sixth-seventh lumbar and lumbar-sacral discs, so a magnetic resonance imaging (MRI) (Siemens Magnetom Essenza 1.5 Tesla, Siemens Healthcare Limited, Camberly, United Kingdom) of the fourth lumbar-caudal spinal segment and CT of the pelvic limbs were performed. Chondrodystrophoid change of the nucleus pulposus of these respective intervertebral discs without compres-

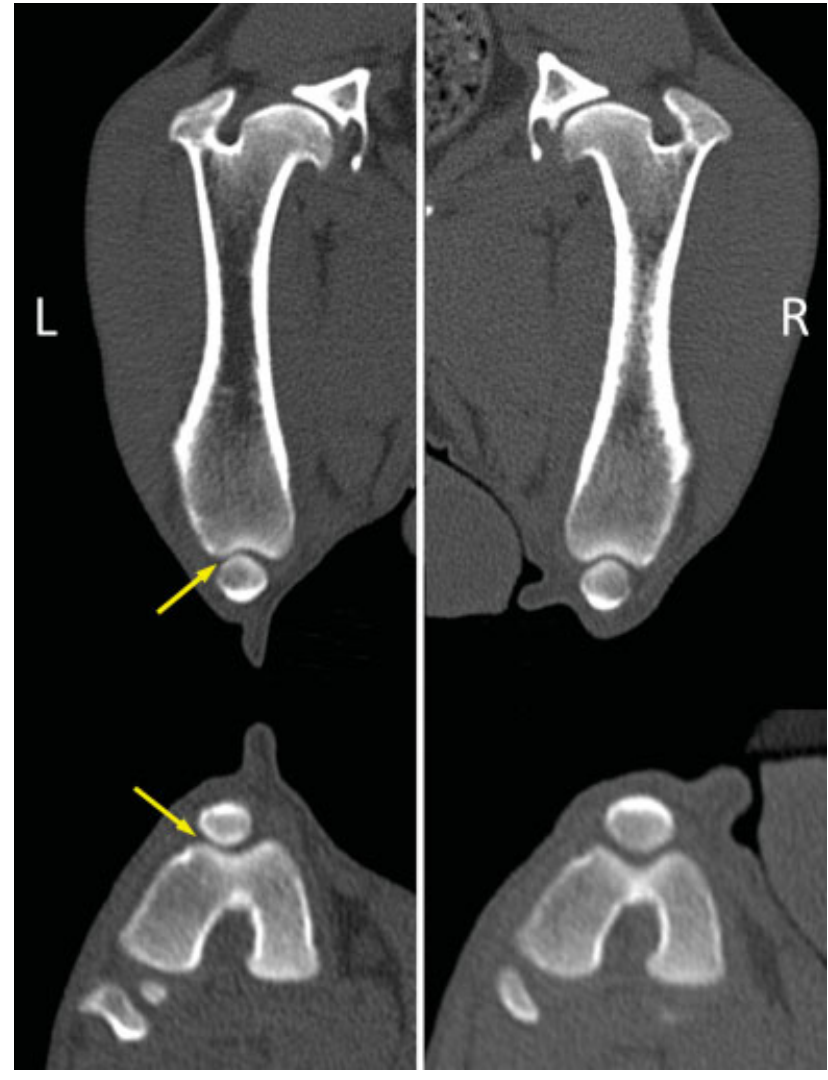

Fig. 2 Dorsal and transverse slice computed tomography (CT) images of the femora and stifles in case 2 . Note that the left lateral patellofemoral joint space is narrowed (yellow arrows).

sion of the spinal cord or nerve roots and mild ventrolateral lumbar spondylosis were present on MRI . Computed tomography of the pelvic limbs revealed eccentric positioning of the left patella in the femoral trochlear sulcus with a widened medial patellofemoral joint space ( - Fig. 2). Surgery on the left stifle was performed. Inspection of the femoral trochlear and patella via a medial parapatellar arthrotomy revealed Outerbridge Grade II cartilage damage on the apex of the lateral trochlear ridge of the femur and Outerbridge Grade IV cartilage damage to the cartilage of the distal half of the patella (-Fig. 3 ). Surgery was performed comprising a block recession sulcoplasty, lateral tibial tuberosity transposition, medial retinacular release and lateral imbrication.

Both dogs were recovered in hospital and pain scored overnight; methadone (Synthadon, Animalcare Ltd., York, United Kingdom) $0.2 \mathrm{mg} / \mathrm{kg}$ being given if they scored above the intervention threshold on the hospital's composite pain score. Both dogs were discharged the day after surgery. The following courses of medication were prescribed for the postoperative period: tramadol (Tramadol Summit Veterinary Pharmaceuticals Ltd., Kidlington, United Kingdom) $5 \mathrm{mg} / \mathrm{kg}$ twice or thrice a day for a week, cephalexin (Cephacare, Animalcare, York, United Kingdom) twice a day for 5 days and meloxicam $0.1 \mathrm{mg} / \mathrm{kg}$ once a day for 2 weeks or as needed. The owners were advised to strictly cage rest the patients postoperatively for 6 weeks, the only exercise being on a short lead for 5 minutes several times a day. 


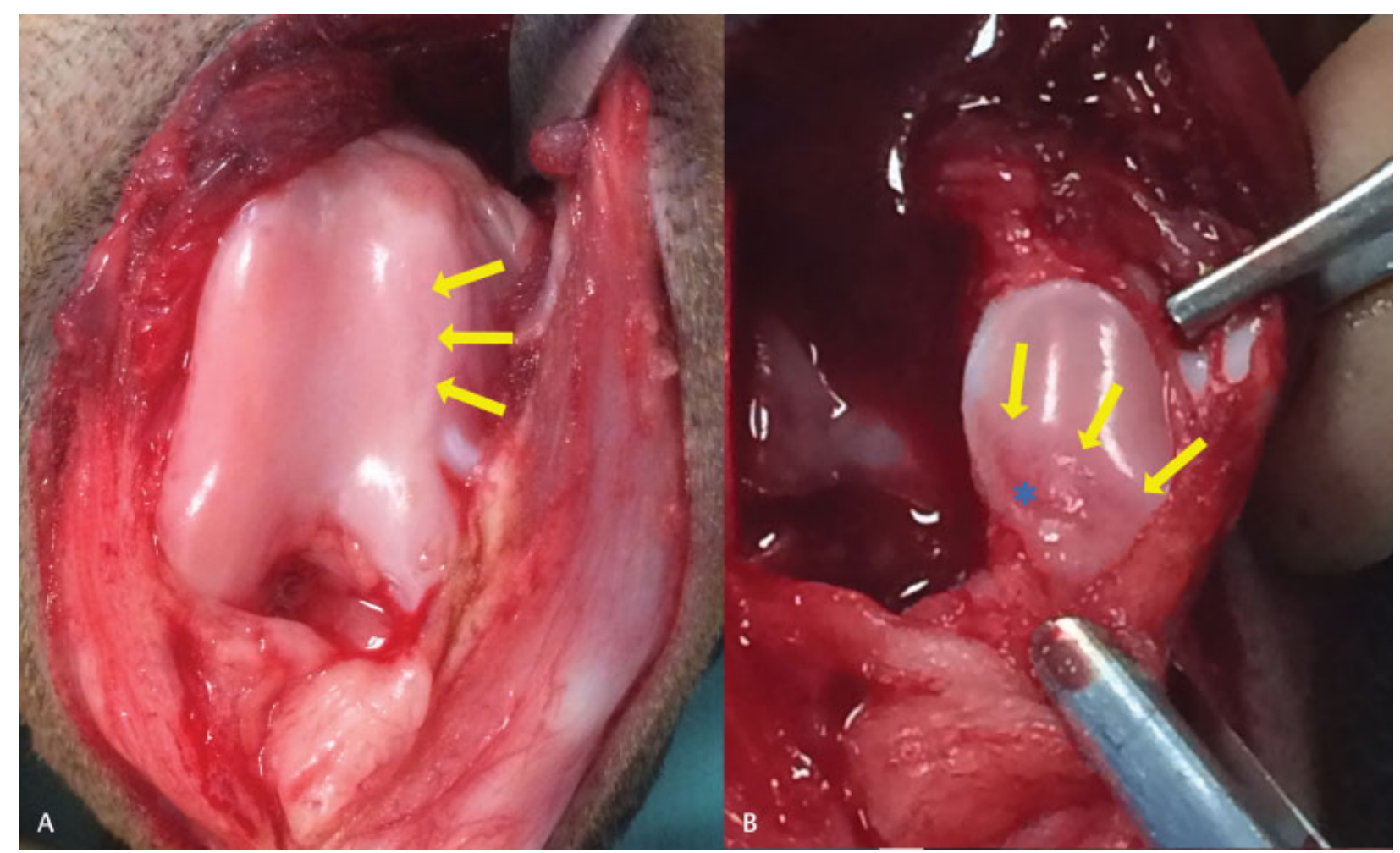

Fig. 3 Intraoperative appearance of (A) trochlear groove, there is evidence of Outerbridge Grade II cartilage damage on the proximal lateral trochlear ridge (yellow arrows), (B) patella, there is evidence of Outerbridge Grade IV cartilage damage affecting the apex pole of the patella (blue asterisk) (yellow arrows delineate the board between grossly normal and eburnated cartilage).

Both patients were examined at 6 weeks postoperatively. Palpation of the affected stifles in both cases revealed the patella to be stable through stifle range of motion. In both cases, the lameness was improved from preoperatively. Radiographs revealed uneventful progression of osteotomy healing. Incrementally, increasing exercise for 6 weeks followed by a return to normal activity was recommended. Telephone follow-up for case 1 (14 months postoperatively) revealed that the dog had been euthanatized for reasons unrelated to the orthopaedic disease previously diagnosed. Telephone followup for case 2 ( 4 months postoperatively) revealed improved function compared with preoperatively but skipping lameness was still occasionally evident in this limb.

\section{Discussion}

This case report has described the clinical, diagnostic imaging and surgical findings of two Dachshunds diagnosed with patellar maltracking as a cause of intermittent pelvic limb lameness. In both cases, clinical examination and radiographs did not reveal any abnormality and diagnosis was only made on the basis of an abnormal patellofemoral joint space on CT scan and subsequent findings at surgery. Due to the small number of cases in this report and limited followup, no conclusions can be drawn on the prevalence or optimum management technique for this condition, but the clinical changes seen serve to highlight that this condition should be included on the list of differential diagnosis for pelvic limb lameness in this breed.
Assessment of the orientation of the patellar long axis (base to apex) relative to the long axis of the femur on threedimensional rendered images from both dogs (-Fig. 4A, B) revealed a lateral deviation of the patellar long axis. The authors speculate that this deviation might result in a congruency mismatch of the femoropatellar joint space with a tendency for focal overload of femoropatellar joint space cartilage medially with the stifle in flexion (-Fig. 4A) and laterally (-Fig. 4B) with the stifle in extension (-Fig. 4C). Focal overload in these regions may explain the topography of the cartilage lesions seen at surgery.

A proposed cause of patellar maltracking in humans is an imbalance between the pull of the vastus medialis and vastus lateralis muscles, which could explain the long axis orientation seen here. Patella alta, trochlear dysplasia and abnormal tensioning in the lateral retinacula, or the medial patellofemoral ligament, are also proposed causes. ${ }^{7,8}$ The preferred management for young human patients with functional patellofemoral syndrome and patellofemoral osteoarthritis is conservative. $^{2,9,10}$ Surgery is indicated in patients who cannot achieve an acceptable level of pain relief. Proximal repairs such as medial reefing and lateral release are suggested to have a lower incidence to long-term osteoarthritis but a higher rate of re-luxations than distal repairs such as tibial tuberosity transfers, so a combination technique is advocated. ${ }^{11}$ This combination technique is akin to what is routinely performed on our patients presenting with patellar luxation.

Persistent, although reduced frequency skipping lameness was evident with case 2 at final follow-up. This could 


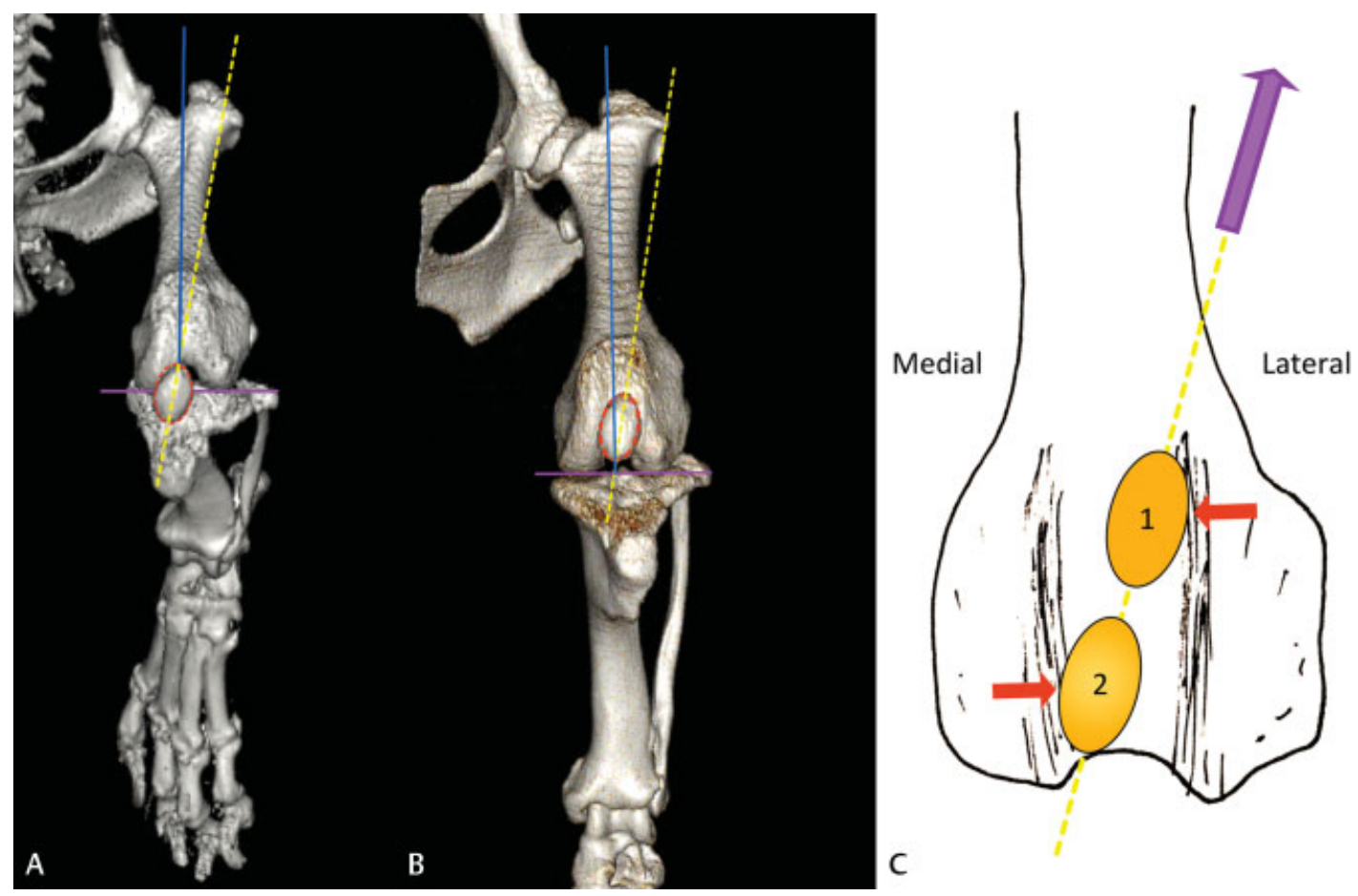

Fig. 4 Three-dimensional (3D) rendered images of the left femur from (A) case 1 and (B) case 2. Assessment of the long axis of the femur (blue line) with reference to a line parallel with the base and apex of the patella (yellow dotted line) are suggestive of a proximolateral to distomedial malalignment of the patella within the patellar groove. The purple line defines the distal extent of the femoral condyle (C) with the patella proximal in the sulcus (1) (i.e. stifle in extension) lateral contact may predominate with a reduced lateral patellofemoral joint space. With the patella distal in the sulcus (2) (i.e. stifle in flexion), medial contact may predominate with a reduced medial patellofemoral joint space. The distal aspect of the femoral condyles is denoted by the purple line.

relate to chondromalacia patellae that was evident at surgery which would be Grade III as defined by Ogilvie-Harris and Jackson. ${ }^{12}$ In that study, humans with patellar maltracking and a concurrent Grade III lesion were less likely to have a good result following surgery.

In summary, patellar maltracking and the associated articular lesions described herein should be included as a differential diagnosis of pelvic limb lameness in the Dachshund. Severe patellar cartilage damage associated with this condition may affect the potential for resolution of lameness with surgical management of this condition.

\section{Conflict of Interest}

None.

\section{Author Contribution}

Neil James Burton contributed the case material for this paper. Both authors contributed to the drafting and revision of the manuscript. Both authors approved the submitted manuscript.

Funding

None.

\section{References}

1 LaBrier K, O'Neill DB. Patellofemoral stress syndrome. Current concepts. Sports Med 1993;16(06):449-459

2 Petersen W, Ellermann A, Gösele-Koppenburg A, et al. Patellofemoral pain syndrome. Knee Surg Sports Traumatol Arthrosc 2014; 22(10):2264-2274
3 Foss KD, Myer GD, Magnussen RA, Hewett TE. Diagnostic differences for anterior knee pain between sexes in adolescent basketball players. J Athl Enhanc 2014;3(01):1814-1820

4 Petersen W, Rembitzki I, Liebau C. Patellofemoral pain in athletes. Open Access J Sports Med 2017;8:143-154

5 O'Neill DG, Meeson RL, Sheridan A, Church DB, Brodbelt DC. The epidemiology of patellar luxation in dogs attending primary-care veterinary practices in England. Canine Genet Epidemiol 2016; 3:4. Doi: 10.1186/s40575-016-0034-0

6 Outerbridge RE, Dunlop JA. The problem of chondromalacia patellae. Clin Orthop Relat Res 1975;(110):177-196

7 Pal S, Draper CE, Fredericson M, et al. Patellar maltracking correlates with vastus medialis activation delay in patellofemoral pain patients. Am J Sports Med 2011;39(03):590-598

8 Pal S, Besier TF, Draper CE, et al. Patellar tilt correlates with vastus lateralis: vastus medialis activation ratio in maltracking patellofemoral pain patients. J Orthop Res 2012;30(06): 927-933

9 Crossley KM, Stefanik JJ, Selfe J, et al. 2016 Patellofemoral pain consensus statement from the 4th International Patellofemoral Pain Research Retreat, Manchester. Part 1: terminology, definitions, clinical examination, natural history, patellofemoral osteoarthritis and patient-reported outcome measures. Br J Sports Med 2016;50 (14):839-843

10 Arazpour M, Notarki TT, Salimi A, Bani MA, Nabavi H, Hutchins SW. The effect of patellofemoral bracing on walking in individuals with patellofemoral pain syndrome. Prosthet Orthot Int 2013;37 (06):465-470

11 Iliadis AD, Jaiswal PK, Khan W, Johnstone D. The operative management of patella malalignment. Open Orthop J 2012; 6:327-339

12 Ogilvie-Harris DJ, Jackson RW. The arthroscopic treatment of chondromalacia patellae. J Bone Joint Surg Br 1984;66(05): 660-665 